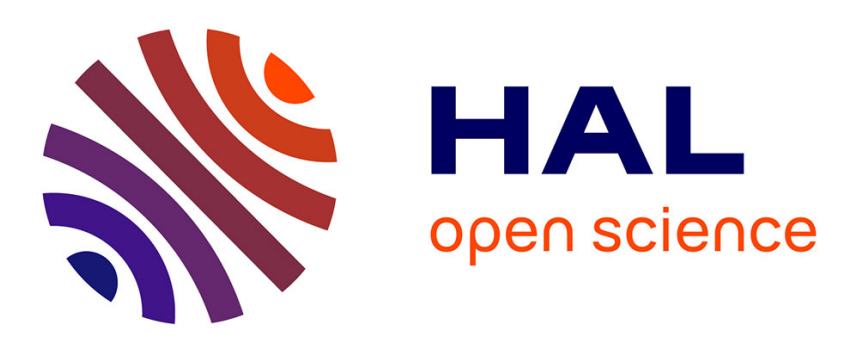

\title{
A New Extended Linear Mixing Model to Address Spectral Variability
}

Miguel Angel Veganzones, Lucas Drumetz, Guillaume Tochon, Mauro Dalla Mura, Antonio Plaza, José M. Bioucas-Dias, Jocelyn Chanussot

\section{- To cite this version:}

Miguel Angel Veganzones, Lucas Drumetz, Guillaume Tochon, Mauro Dalla Mura, Antonio Plaza, et al.. A New Extended Linear Mixing Model to Address Spectral Variability. WHISPERS 2014 - 6th Workshop on Hyperspectral Image and Signal Processing: Evolution in Remote Sensing, Jun 2014, Lausanne, Switzerland. pp.n/c. hal-01010424

\section{HAL Id: hal-01010424 https://hal.science/hal-01010424}

Submitted on 19 Jun 2014

HAL is a multi-disciplinary open access archive for the deposit and dissemination of scientific research documents, whether they are published or not. The documents may come from teaching and research institutions in France or abroad, or from public or private research centers.
L'archive ouverte pluridisciplinaire $\mathbf{H A L}$, est destinée au dépôt et à la diffusion de documents scientifiques de niveau recherche, publiés ou non, émanant des établissements d'enseignement et de recherche français ou étrangers, des laboratoires publics ou privés. 


\title{
A NEW EXTENDED LINEAR MIXING MODEL TO ADDRESS SPECTRAL VARIABILITY
}

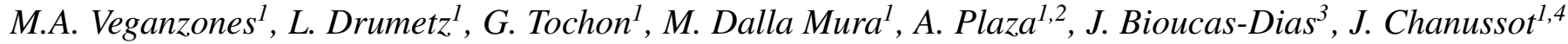 \\ ${ }^{1}$ GIPSA-lab, Grenoble-INP, Saint Martin d'Hères, France \\ ${ }^{2}$ Hyperspectral Computing Laboratory, University of Extremadura (UEX), Cáceres, Spain \\ ${ }^{3}$ Instituto de Telecomunicações and Instituto Superior Técnico, Universidade de Lisboa, Lisbon, Portugal \\ ${ }^{4}$ Faculty of Electrical and Computer Engineering, University of Iceland, Reykjavik, Iceland
}

\begin{abstract}
Spectral variability is a phenomenon due, to a grand extend, to variations in the illumination and atmospheric conditions within a hyperspectral image, causing the spectral signature of a material to vary within a image. Data spectral fluctuation due to spectral variability compromises the linear mixing model (LMM) sum-to-one constraint, and is an important source of error in hyperspectral image analysis. Recently, spectral variability has raised more attention and some techniques have been proposed to address this issue, i.e. spectral bundles. Here, we propose the definition of an extended LMM (ELMM) to model spectral variability and we show that the use of spectral bundles models the ELMM implicitly. We also show that the constrained least squares (CLS) is an explicit modelling of the ELMM when the spectral variability is due to scaling effects. We give experimental validation that spectral bundles (and sparsity) and CLS are complementary techniques addressing spectral variability. We finally discuss on future research avenues to fully exploit the proposed ELMM.
\end{abstract}

Index Terms - Spectral unmixing, extended linear mixing model, spectral bundles, sparsity, CLS.

\section{INTRODUCTION}

Hyperpectral unmixing is one of the most important and widely used techniques in hyperspectral image analysis. It consists in decomposing the hyperspectral image into a set of spectral signatures corresponding to macroscopically pure materials, named endmembers, and a set of cover proportions comprised in a fractional abundance matrix. In the Linear Mixing Model (LMM) [1], hyperspectral data are modelled as a linear combination of the endmembers weighted by their fractional abundances. According to the LMM definition, data lie into a simplex whose vertexes are defined by the endmembers.

However, real data present fluctuations that make them lie outside the simplex. These fluctuations are due to variable illumination and atmospheric conditions, causing the spectral signature of a material to vary within the image [2]. This is known as the spectral variability issue and, not addressing it may introduce errors that propagate throughout the hyperspectral image analysis process. Recently, authors in [2] presented an overview of the techniques to address

Miguel A. Veganzones was supported in part by ERC AdG-2013-320594 DECODA and by the Delegation Generale de l'Armement (DGA) under contract PDE2012600079. Prof. Bioucas was supported by the Portuguese Science and Technology Foundation under Projects PEst-OE/EEI/LA0008/2013 and PTDC/EEI-PRO/1470/2012. spectral variability which could be roughly divided in two: a) representing the spectral variability by spectral bundles and, b) modelling the variability by means of probabilistic distributions.

Here, we propose an extension to the LMM, called Extended LMM (ELMM), to face spectral variability. This ELMM is based on the model defined in [3] and relies on the definition of a pixeldependent function, called the spectral variability function, that defines how a given endmember varies along the image. We show that the use of spectral bundles is a methodology to implicitly model this spectral variability function. We further prove, that given some assumptions, the Constrained Least Squares (CLS) unmixing process is an explicit model of this function. We overview these two approaches, i.e., spectral bundles and CLS unmixing. In addition, we study the use of sparsity constraints on the light of the proposed ELMM and we discuss further research avenues to enhance the modelling of spectral variability using the ELMM. We experimentally show that both, spectral bundles and CLS, are complementary techniques to address spectral variability. We also show that adding sparsity terms to the unmixing optimization process is helpful in combination with the spectral bundles, and that CLS is inherently enforcing sparsity as some authors have pointed out before [4,5].

The remainder of the paper is as follows: Sec. 2 introduces the ELMM. Sec. 3 overviews spectral bundles, sparsity and CLS on the light of the proposed ELMM. Sec. 4 provides experimental insight. Sec. 5 presents a discussion on future research avenues to further exploit the proposed ELMM, and finally we give some conclusion remarks in Sec. 6.

\section{THE EXTENDED LINEAR MIXING MODEL}

Let $\mathbf{E}=\left[\mathbf{e}_{1}, \ldots, \mathbf{e}_{m}\right]$ denote the pure endmember signatures in the hyperspectral image, where each $\mathbf{e}_{i} \in \mathbb{R}^{q}$ is a $q$-dimensional vector. The LMM models a hyperspectral vector $\mathbf{r}_{j}$ at $j$-th pixel as follows:

$$
\mathbf{r}_{j}=\mathbf{s}_{j}+\mathbf{n}_{j}=\sum_{i=1}^{m} \mathbf{e}_{i} \phi_{i j}+\mathbf{n}_{j},
$$

where $\mathbf{r}_{j}$ is given by the sum of the pixel's signal $\mathbf{s}_{j}$ and an independent additive noise component $\mathbf{n}_{j}$; and, $\phi_{j}$ is the $m$-dimensional vector of fractional per-pixel abundances. The constraints on the fractional abundances are given by:

$$
\begin{gathered}
\text { (ANC) } \quad \phi_{i j} \geq 0, \forall i, \forall j, \\
\text { (ASC) } \quad \sum_{i=1}^{m} \phi_{i j}=1, \forall j .
\end{gathered}
$$

Dismissing the noise, the hyperspectral pixels in the LMM (1) lie inside a simplex whose vertexes are the endmembers and their frac- 
tional abundances can be estimated by an optimization process

$$
\hat{\boldsymbol{\phi}}_{j}=\arg \min _{\boldsymbol{\phi}_{j} \geq \mathbf{0}}\left\|\mathbf{r}_{j}-\sum_{i=1}^{m} \mathbf{e}_{i} \phi_{i j}\right\|^{2}
$$

subject $\sum_{i=1}^{m} \phi_{i j}=1$.

However, in real scenarios, spectral variabilities seriously affect the LMM. These variabilities can be modelled as a pixel-dependent function of the endmembers, $f: \mathbb{R}^{q} \rightarrow \mathbb{R}^{q}$, so the LMM in (1) is redefined as:

$$
\mathbf{r}_{j}=\sum_{i=1}^{m} f_{j}\left(\mathbf{e}_{i}\right) \phi_{i j}+\mathbf{n}_{j} .
$$

The model in (5) will be hereafter named as the Extended LMM (ELMM).

\section{ASSESSING SPECTRAL VARIABILITY}

The use of the ELMM (5) to model spectral variability can be addressed in two ways: i) by implicitly encompassing the spectral variability in the scene and, ii) by explicitly modelling the variability function, $f_{j}\left(\mathbf{e}_{i}\right)$, in (5). The use of spectral bundles is a solution to the former while, under some assumptions, the partially constrained least squares (CLS) solution to (1) is a solution to the latter one, as we prove below.

\subsection{Spectral bundles and sparsity}

Spectral bundles are sets of spectral signatures that implicitly represent spectral variability. A given spectral bundle:

$$
\mathbf{B}_{i}=\left\{\tilde{\mathbf{e}}_{i k}\right\}_{k=1}^{K},
$$

is composed of a set of $K$ spectral signatures, $\left\{\tilde{\mathbf{e}}_{i k}\right\}$, that account for the spectral variability of an endmember, $\mathbf{e}_{i}$. The spectral bundles approach does not explicitly model the variability function, $f_{j}\left(\mathbf{e}_{i}\right)$, but it works as a collection of outcomes of the variability function that implicitly represent the spectral variability along the whole image.

In order to build the spectral bundles from the data, $\mathcal{B}=$ $\bigcup_{i=1}^{m} \mathbf{B}_{i}$, one can run an endmember induction algorithm over multiple subsets of the data set obtained by sampling with replacement [6]. An alternative approach consists in a local definition of the bundles by using a sliding window [7]. Anyway, the spectral bundles are usually a large collection of spectra. Therefore, the spectral unmixing of the data by spectral bundles is usually accompanied with a sparsity constraint which enforces that only a few spectral signatures in the bundle take positive abundance values:

$$
\hat{\boldsymbol{\phi}}_{j}=\arg \min _{\boldsymbol{\phi}_{j} \geq \mathbf{0}}\left\|\mathbf{r}_{j}-\sum_{l=1}^{L} \tilde{\mathbf{e}}_{l} \phi_{l j}\right\|_{2}^{2}+\tau\left\|\boldsymbol{\phi}_{j}\right\|_{1},
$$

subject $\sum_{i=1}^{m} \phi_{i j}=1$, where $\tau \geq 0$ is a regularization parameter and $\|\cdot\|_{p}$ denotes the $p$-norm.

\subsection{Partially constrained least squares (CLS)}

Since spectral variability is dominated by scaling factors [8], we adopt the following spectral variability function:

$$
f_{j}\left(\mathbf{e}_{i}\right)=\lambda_{i j} \mathbf{e}_{i},
$$

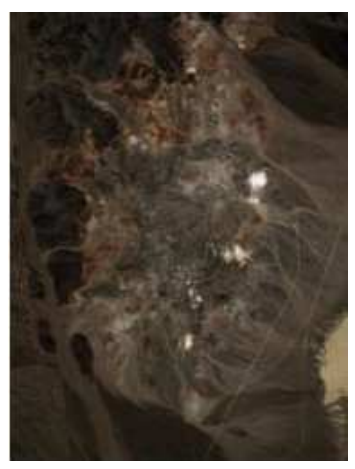

Fig. 1. A false color representation of the Cuprite hyperspectral data set.

where $\lambda_{i j} \geq 0$ denotes a scaling factor. Substituting (8) into the ELMM (5):

$$
\mathbf{r}_{j}=\sum_{i=1}^{m} \mathbf{e}_{i} \lambda_{i j} \phi_{i j}+\mathbf{n}_{j} .
$$

In (9), the hyperspectral pixels lie inside the positive hypercone defined by the endmembers. The CLS is an approximate solution to (9) which is solved by the following optimization problem:

$$
\hat{\boldsymbol{a}}_{j}=\arg \min _{\boldsymbol{a}_{j} \geq \mathbf{0}}\left\|\mathbf{r}_{j}-\sum_{i=1}^{m} \mathbf{e}_{i} a_{i j}\right\|^{2},
$$

The estimated weighting factors incorporate the information from the spectral abundances, $\phi_{i j}$ and the scaling factors, $\lambda_{i j}$, that is:

$$
\hat{a}_{i j}=\hat{\lambda}_{i j} \hat{\phi}_{i j} .
$$

In order to retrieve both informations from (11), it is possible to assume that the scaling factor is the same for all the endmembers, $\lambda_{i j}=\lambda_{j}, \forall i$, and then, estimate it as:

$$
\hat{\lambda}_{j}=\sum_{i=1}^{m} \hat{a}_{i j} .
$$

This makes sense only when the variability is due to illumination/topographic factors [9]. Then, the fractional abundances can be obtained by normalizing the vector of weighting factors by the estimated scaling factor, $\hat{\phi}_{i j}=\hat{a}_{i j} / \hat{\lambda}_{j}$, so the ASC (3) is fulfilled. The CLS is a simple approach to address the spectral variability assuming this is given by a scaling factor that affects equally to all the endmembers present in a pixel.

\section{EXPERIMENTAL VALIDATION}

Here we intent to experimentally get an insight on the capability of the spectral bundles, sparsity and CLS methodologies to address the spectral variability issue. We test the aforementioned approaches on the Cuprite data set [10] (see Fig. 1). The scene was taken by the NASA's AVIRIS sensor and covers the Cuprite mining district in western Nevada, USA.

We made use of the Vertex Component Analysis (VCA) algorithm [3] to induce the endmembers from the data. We built three sets of endmembers: the classical approach running the VCA over the whole dataset, denoted as $\mathbf{E}$, and two spectral bundles using the 

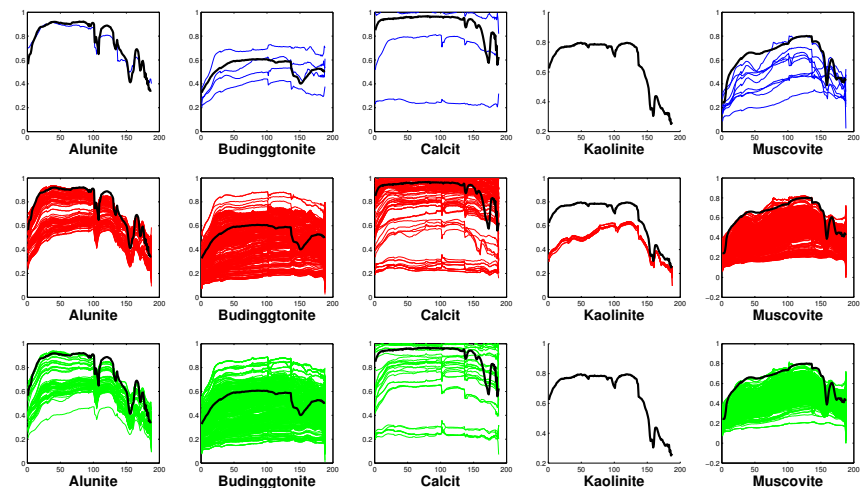

Fig. 2. Spectral signatures of the set of endmembers $\mathbf{E}$ (blue) and the two bundle sets, $\mathbf{R}$ (red) and $\mathbf{W}$ (green), and their assignation to one of the five ground truth endmembers (black).

sampling with replacement and the sliding window methods, respectively denoted as $\mathbf{R}$ and $\mathbf{W}$. In order to solve the unmixing optimization problems, we use the SUnSAL algorithm [11], which is an instance of the C-SALSA methodology introduced in [12] to effectively solve a large number of constrained LS problems sharing the same matrix system.

Fig. 2 shows the spectra of the set of endmembers $\mathbf{E}$ and the two spectra bundles, $\mathbf{R}$ and $\mathbf{W}$. We assigned each spectral signature to the closest spectral signature of the materials known to be present in the scene, in terms of spectral angle distance. The spectral signatures has been obtained from the USGS spectral library. In total, the set $\mathbf{E}$ contains 17 endmembers while the bundles $\mathbf{R}$ and $\mathbf{W}$ contain 1669 and 1353 spectral signatures respectively. It can be noted that the bundle sets cover an abroad spectral variability range. The Kaolinite endmember seems difficult to retrieve. This could be due to scarcity of the material or to its mixture with Alunite which presents a similar spectral pattern. Nevertheless, the sampling with replacement bundle is able to induce a few spectral signatures corresponding to this endmember.

Fig. 3 shows the average reconstruction errors obtained for the three different endmember induction approaches combined to the FCLS or CLS unmixing, with or without sparsity, where $t$ denotes the value assigned to the sparsity factor. The CLS approach outperforms the FCLS approach as it was expected, and it is specially remarkable for the $\mathbf{E}$ set, where there is no implicit information about spectra variability in the form of a bundle. The use of spectral bundles also yields to a great improvement of the reconstruction errors. Sparsity only works if there is enough spectral variability, that is, when the spectral bundles are used, but in these cases helps to obtain a better reconstruction. However, setting the sparsity factor to a high value $\left(t=10^{-2}\right)$, has a counter-effect yielding to poor reconstruction results. Finally, the sliding window approach obtains the best results, showing that the use of locality in the bundles construction is meaningful. In Fig. 4 it is shown the distributions of the number of spectral signatures that take positive abundance values $(\phi>0)$ for each approach. This strengthens the above idea that, in order to enforce sparsity, it is necessary to model the spectral variability by the set of endmembers. Also, it can be shown that the CLS approach indirectly enforces some sparsity as it was noted in $[4,5]$.

The experimental results reinforces the notion that both spectral bundles and CLS are valuable approaches to address spectral variability, and that sparsity could help as well when it is combined with
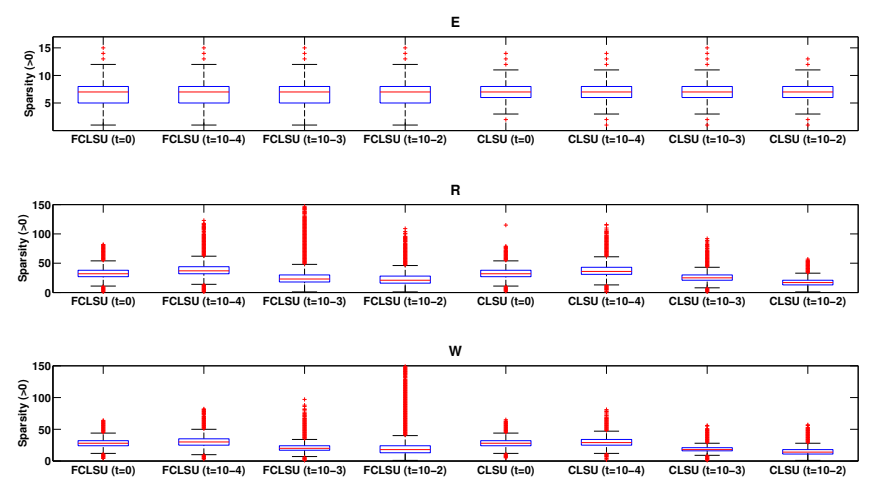

Fig. 4. Active endmembers

the spectral bundles, that is, when there is enough implicit spectral variability in the set of spectra used to solve the unmixing optimization problem.

\section{DISCUSSION}

The proposed ELMM (5) is a natural extension of the LMM (1) in order to model spectral variability. The ELMM is based on the definition of a spectral variability function that expresses the pixeldependent variability of each endmember. Here, we have discussed spectral bundles and CLS techniques on the light of this extended model, and we have shown how they implicitly or explicitly model it. Following, we discuss some avenues for further work: 1) The spectral bundles implicitly model $f_{j}\left(\mathbf{e}_{i}\right)$, but it could be possible to use the spectral bundles to estimate $f_{j}\left(\mathbf{e}_{i}\right)$ from the data, i.e. using parametric models. 2) The CLS approach makes use of strong assumptions to solve $f_{j}\left(\mathbf{e}_{i}\right)$. Further work will make emphasis in more relaxed assumptions that will require additional information, i.e. spatial smoothness of the scaling factor across the image. 3) In the text, in order to keep the explanation as clear as possible, it has been avoided to define the spectral variability function in terms of the spectral bands. However, it is reasonable that the spectral variability varies according to the spectral wavelengths $(\omega): f_{j}^{\omega}\left(e_{i}^{\omega}\right)$. However, this adds an extra complexity to the model. 4) In this paper we have focused on the use of deterministic sets (endmembers and bundles) to define the spectral information.

\section{CONCLUSIONS}

We have shown on the light of a new Extended LMM that spectral bundles and CLS are two different ways to model the spectral variability in a hyperspectral image. We also have provided experimental results that highlights the performance of these approaches respect to the classical approach based on a set of endmembers. The use of sparsity has been also considered and shown that it plays a role only when spectral variability is included in the set of spectral signatures. Finally, we have discussed future research avenues in order to further exploit the proposed Extended LMM.

\section{REFERENCES}

[1] N. Keshava and J.F. Mustard, "Spectral unmixing," Signal Processing Magazine, IEEE, vol. 19, no. 1, pp. 44-57, 2002. 


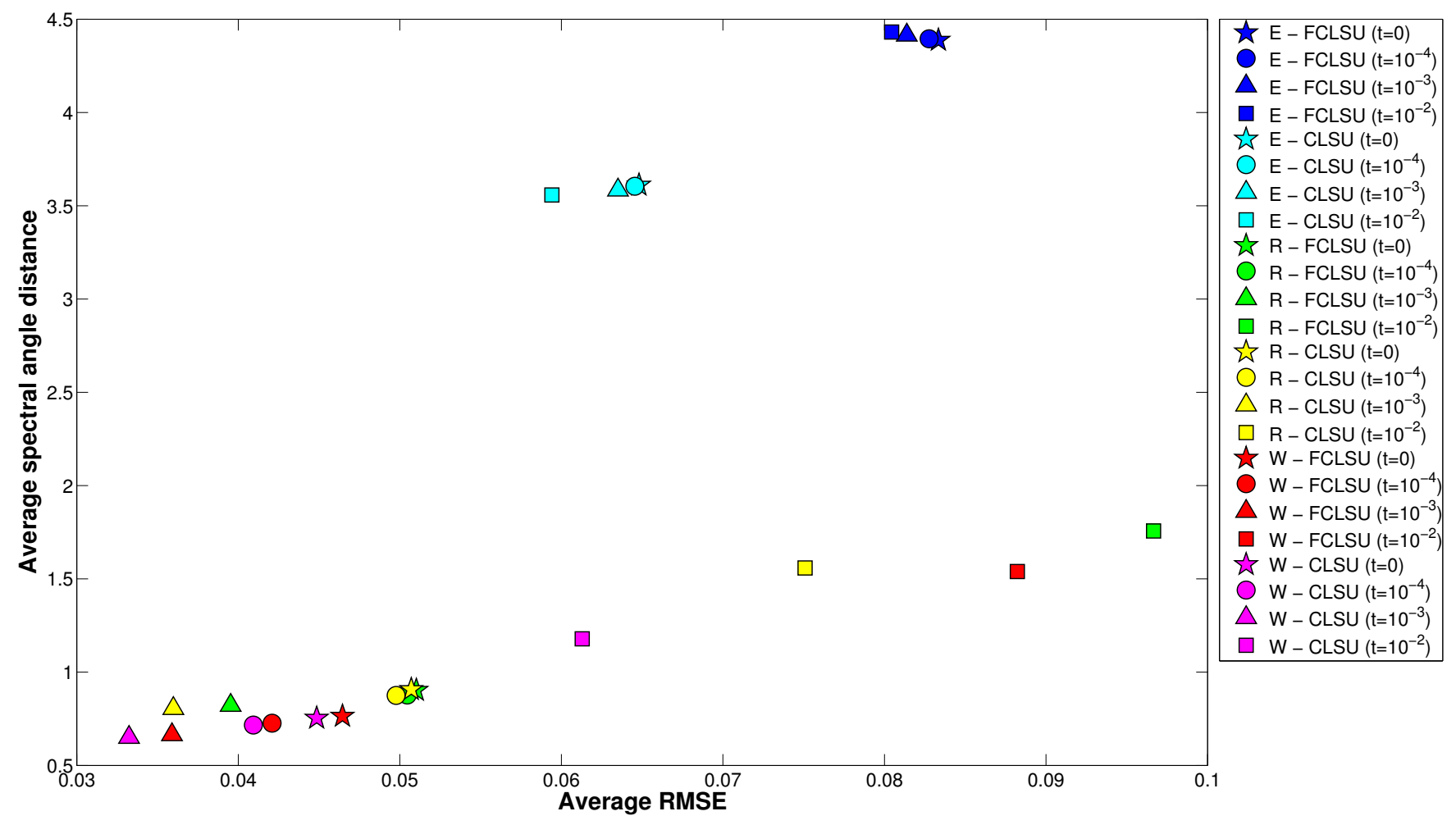

Fig. 3. Average reconstruction errors of the E+FCLSU (blue), E+CLSU (cyan), R+FCLSU (green), E+CLSU (yellow), W+FCLSU (red) and $\mathbf{W}+\mathrm{CLSU}$ (magenta) approaches. Sparsity, $t=\left[0,10^{-4}, 10^{-3}, 10^{-2}\right]$, is depicted in geometrical shapes.

[2] A. Zare and K.C. Ho, "Endmember variability in hyperspectral analysis: Addressing spectral variability during spectral unmixing," IEEE Signal Processing Magazine, vol. 31, no. 1, pp. 95-104, Jan 2014.

[3] J.M.P. Nascimento and J.M. Bioucas-Dias, "Vertex component analysis: a fast algorithm to unmix hyperspectral data," IEEE Transactions on Geoscience and Remote Sensing, vol. 43, no. 4, pp. 898 - 910, april 2005.

[4] A.M. Bruckstein, M. Elad, and M. Zibulevsky, "On the uniqueness of nonnegative sparse solutions to underdetermined systems of equations," IEEE Transactions on Information Theory, vol. 54, no. 11, pp. 4813-4820, 2008.

[5] M.-D. Iordache, J.M. Bioucas-Dias, and A. Plaza, "Sparse unmixing of hyperspectral data," IEEE Transactions on Geoscience and Remote Sensing, vol. 49, no. 6, pp. 2014-2039, June 2011.

[6] B. Somers, M. Zortea, A. Plaza, and G.P. Asner, "Automated extraction of image-based endmember bundles for improved spectral unmixing," IEEE Journal of Selected Topics in Applied Earth Observations and Remote Sensing, vol. 5, no. 2, pp. 396408, April 2012.

[7] M.A. Goenaga, M.C. Torres-Madronero, M. Velez-Reyes, S.J. Van Bloem, and J.D. Chinea, "Unmixing analysis of a time series of hyperion images over the guanica dry forest in puerto rico," IEEE Journal of Selected Topics in Applied Earth Observations and Remote Sensing, vol. 6, no. 2, pp. 329-338, April 2013.
[8] J.M. Bioucas-Dias, A. Plaza, N. Dobigeon, M. Parente, Qian $\mathrm{Du}, \mathrm{P}$. Gader, and J. Chanussot, "Hyperspectral unmixing overview: Geometrical, statistical, and sparse regression-based approaches," IEEE Journal of Selected Topics in Applied Earth Observations and Remote Sensing, vol. 5, no. 2, pp. 354-379, April 2012.

[9] J.M.P. Nascimento and J.M. Bioucas Dias, "Does independent component analysis play a role in unmixing hyperspectral data?," IEEE Transactions on Geoscience and Remote Sensing, vol. 43, no. 1, pp. 175-187, Jan 2005.

[10] R.O. Green, M.L. Eastwooda, C.M. Sarturea, T.G. Chriena, M. Aronssona, B.J. Chippendalea, J.A. Fausta, B.E. Pavria, C.J. Chovita, M. Solisa, M.R. Olaha, and O. Williamsa, "Imaging spectroscopy and the airborne visible/infrared imaging spectrometer (aviris)," Remote Sensing of Environment, vol. 65, no. 3, pp. $227-248,1998$.

[11] J.M. Bioucas-Dias and M.A.T. Figueiredo, "Alternating direction algorithms for constrained sparse regression: Application to hyperspectral unmixing," in 2010 2nd Workshop on Hyperspectral Image and Signal Processing: Evolution in Remote Sensing (WHISPERS). IEEE, 2010, pp. 1-4.

[12] M.V. Afonso, J.M. Bioucas-Dias, and M.A.T. Figueiredo, "An augmented lagrangian approach to the constrained optimization formulation of imaging inverse problems," IEEE Transactions on Image Processing, vol. 20, no. 3, pp. 681-695, 2011. 